\title{
Dialysate interleukin-6 predicts increasing peritoneal solute transport rate in incident peritoneal dialysis patients
}

Yeoungjee Cho ${ }^{1,2,3}$, David W Johnson ${ }^{1,2,3^{*}}$, David A Vesey ${ }^{1,2,3}$, Carmel M Hawley ${ }^{1,2,3}$, Elaine M Pascoe ${ }^{2}$, Margaret Clarke ${ }^{4}$, Nicholas Topley ${ }^{5}$ and on behalf of the balANZ Trial Investigators

\begin{abstract}
Background: Repeated exposure to peritoneal dialysis (PD) solutions contributes to cumulative intraperitoneal inflammation and peritoneal injury. The present study aimed to explore the capacity of dialysate interleukin-6(IL-6) to a) predict peritoneal membrane function and peritonitis in incident PD patients, and b) to evaluate the influence of neutral pH, low glucose degradation product (GDP) PD solution on dialysate IL-6 levels.

Methods: The study included 88 incident participants from the balANZ trial who had completed 24-months of follow-up. Change in peritoneal solute transport rate (PSTR) and peritonitis were primary outcome measures, and the utility of IL-6 and IL-6 appearance rate (IL-6 AR) in predicting these outcomes was analyzed using multilevel linear regression and Cox proportional hazards models, respectively. Sensitivity analyses were performed by analyzing outcomes in a peritonitis-free cohort $(n=56)$.

Results: Dialysate IL-6 concentration significantly increased from baseline to 24 months (mean difference $19.07 \mathrm{pg} / \mathrm{mL}$; $P<0.001)$ but was not affected by the type of PD solution received $(P=0.68)$. An increase in PSTR from baseline was associated with higher levels of $\mathrm{IL}-6(P=0.004)$, the use of standard solutions $(P=0.005)$ and longer PD duration $(P<0.001)$. Baseline IL-6 level was not associated with a shorter time to first peritonitis (adjusted hazard ratio 1.00, $95 \% \mathrm{Cl} 0.99-1.00, P=0.74)$. Analysis of IL-6 AR as well as sensitivity analyses in a peritonitis-free cohort yielded comparable results.

Conclusion: Dialysate IL-6 concentration increased with longer PD duration and was a significant, independent predictor of PSTR. The use of biocompatible PD solutions exerted no significant effect on dialysate IL-6 levels but did abrogate the increase in PSTR associated with standard PD solutions. This is the first study to examine the impact of biocompatible solutions on the utility of IL-6 in predicting PSTR and peritonitis.
\end{abstract}

Keywords: Biocompatible, Glucose degradation products, Interleukin-6, Peritoneal dialysis, Peritoneal solute transport rate, Peritonitis

\section{Background}

The peritoneum is composed of a monolayer of mesothelial cells resting upon a thin basement membrane [1,2]. Continuous exposure to conventional peritoneal dialysis (PD) solutions contributes to progressive peritoneal injury, is an important source of local inflammation that can result in

\footnotetext{
* Correspondence: david.johnson2@health.qld.gov.au

'Department of Renal Medicine, Princess Alexandra Hospital, Brisbane, Australia

${ }^{2}$ School of Medicine, University of Queensland, Brisbane, Australia Full list of author information is available at the end of the article
}

adverse functional outcomes, such as higher peritoneal solute transport rate (PSTR), a widely accepted risk factor for mortality and technique failure in PD patients [3-5].

Interleukin-6 (IL-6) is an immuno-modulatory cytokine that plays a critical role in many innate and acquired inflammatory processes [6]. It is secreted in large quantities by peritoneal mesothelial cells in response to inflammatory stimuli and modulated by exposure to PD solutions [7]. Interest in IL-6 and its role in intraperitoneal inflammation in PD patients is increasing as a result of the link from single centre studies to changes in PSTR,

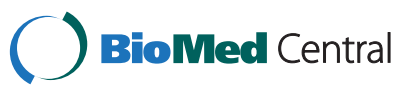


data that has now been corroborated by recent analysis from the large multicenter GLOBAL study [8-10]. To date there are no data on the impact of biocompatible PD solution use on IL-6 levels and its relationship to PSTR. Improvement in peritoneal membrane morphology from the use of more biocompatible solutions, characterized by neutral $\mathrm{pH}$ with lowered glucose degradation product (GDP), has been suggested previously $[11,12]$. Therefore, its use may lead to changes in the intraperitoneal inflammatory milieu with potential benefits to peritoneal membrane function and improved host defence against peritonitis. Although a number of randomized controlled trials (RCT) have reported comparable dialysate IL-6 levels from biocompatible and standard solutions use [13-16], these studies were limited by open and variable prescription, large drop out rates (>20\%) [16], cross-over study designs with the risk of carry-over effect [14], risk of bias from including biocompatible solutions with variable GDP levels in the treatment group [15], and a lack of accounting for the confounding effect of peritonitis [13-16]. The utility of dialysate IL- 6 as a potential predictor of adverse peritoneal membrane function has not been studied within an RCT setting.

The aims of the present study were to describe the trend in dialysate IL- 6 concentration and IL- 6 appearance rate (IL-6 AR) in incident PD patients, to explore the utility of dialysate IL- 6 as a predictor of PSTR and peritonitis in this patient group and to evaluate the impact of neutral $\mathrm{pH}$, low GDP PD solutions on these outcomes.

\section{Methods}

\section{Study design}

Data were obtained from the participants of the balANZ trial [17]. Detailed description of the study design and methodology has been previously published [18], as have the results of the main primary and secondary analyses $[17,19,20]$. The trial was registered with the Australian New Zealand Clinical Trials Registry (ACTRN12606000044527). The study protocol was approved by the ethics committees at Princess Alexandra Hospital Research Ethics Committee as well as all other participating units (see Appendix for list of hospitals). All patients provided written informed consent prior to trial participation, including consent to biomarker studies using stored samples. Incident, adult PD patients who had commenced dialysis for the first time within the preceding 90 days with both a residual measured glomerular filtration rate $($ GFR $) \geq 5 \mathrm{ml} / \mathrm{min} / 1.73 \mathrm{~m}^{2}$ and a measured urine volume $\geq 400 \mathrm{ml} /$ day at enrolment were included in the study. Pregnant or breastfeeding patients, individuals expected to die within 12 months, patients participating in trials targeting residual renal function in PD or those with a significant cancer history in the past 5 years, acute infection at enrolment, contra-indications to $\mathrm{PD}$, any physical or mental disorder that appreciably hampered study protocol compliance or known or suspected allergy to trial product or related products were excluded. Of the 185 participants of the balANZ trial, 88 participants who had completed 24-month follow-up (Balance $^{\curvearrowleft} \mathrm{n}=42$; Stay.Safe $^{\oplus} \mathrm{n}=46$ ) with at least one PD effluent (PDE) sample stored during trial participation were included in the present investigation.

\section{IL-6}

PDE samples were collected at baseline, 12, and 24 month visits. PDE was initially stored in a $-20^{\circ} \mathrm{C}$ or $-80^{\circ} \mathrm{C}$ freezer locally, then transported frozen to a central storage facility and kept at $-80^{\circ} \mathrm{C}$. Samples were thawed once only during the aliquoting process prior to analysis. IL- 6 was measured by an electrochemiluminescence immunoassay technique using the manufacturer's protocols. 96-well plates measuring IL-6 were analysed on a Sector Imager $6000^{\circ}$ (Mesoscale Discovery [MSD], Gaithusburg, MD, USA). Samples and standards were analysed in duplicates with a maximum tolerated coefficient of variation $(\mathrm{CV})$ of $20 \%$. The lower level of detection limit was $0.07 \mathrm{pg} / \mathrm{mL}$. No inter-assay CV was determined as all samples from an individual were run at the same time to minimize between-assay variability.

\section{Clinical outcomes}

The clinical outcome measures were: 1) PSTR defined as 4 hour dialysate:plasma creatinine ratio $\left(\mathrm{DP}_{\mathrm{cr} 4 \mathrm{~h}}\right)$ measured during the peritoneal equilibration test (PET), and 2) episodes of PD-related peritonitis during study participation.

\section{Calculations}

IL-6 AR was calculated by multiplying the PDE IL-6 concentrations and drained volume, which was then divided by the dwell time and expressed as picograms per minute. To account for individual variation in baseline IL- 6 concentrations and peritoneal functional capacity, the change in IL-6, IL-6 AR, and PSTR were calculated for month 12 (month 12 - baseline); and month 24 (month 24 - baseline). Baseline values of PSTR were measured at month 1 .

\section{Statistical analyses}

Results were expressed as frequencies (percentages) for categorical variables, mean \pm standard deviation (SD) for continuous normally distributed variables, and median (interquartile range) for continuous non-normally distributed variables. Differences between groups on baseline characteristics were analysed by $\chi^{2}$ test for categorical data, t-test or Mann-Whitney $U$ test for continuous data, as appropriate. The overall trend in IL- 6 concentrations and IL- 6 AR were analysed by fitting a multilevel linear regression model to the logtransformed IL-6 data (or IL-6 AR). Categorical time 
(i.e. baseline, 12, 24 months) was included as a fixedeffect and random intercepts and slopes were added to allow for repeated measurements over time. To evaluate the differences between the two treatment groups on IL-6, PD solution type (Biocompatible vs. Control) and the interaction between solution type and time were subsequently added to the model as fixed effects. If the interaction term was not statistically significant, the unconditional effects of PD solution type on IL-6 would be retained and the interaction term was removed from the final model. In addition, the baseline IL- 6 or IL- 6 AR were explored as potential predictors of change in their respective post-baseline levels. The IL-6 and IL-6 AR data were log transformed due to their non-normal distributions. As a sensitivity analysis, the role of loss of residual renal function (calculated by GFR at relevant month - baseline value) on IL- 6 concentrations over time was explored as a time-varying covariate in the multilevel linear regression model.

To determine whether IL- 6 was associated with changes in PSTR, a multilevel linear regression model was fitted. Clinically recognized risk factors that affect PSTR (such as time on PD, racial origin, age, body mass index [BMI], male gender) [21,22] and randomly assigned type of PD solution [19] were included as fixed-effects in an initial full model. Variables with statistically non-significant effects in the full model were removed from the final model. The fit of the final model was checked against the full model using the likelihood ratio test. Any significant interaction identified was added to the model. Due to the potential bias introduced by peritonitis-related increase in IL-6 and PSTR, a sensitivity analysis in a subgroup who were peritonitisfree was performed (biocompatible $n=34$; control $n=22$ ).

Time to first peritonitis was analyzed by a multivariable Cox proportional hazards model. Baseline IL-6, PD solution type, age, sex, racial origin, BMI, diabetes mellitus, PD modality (automated PD/continuous ambulatory PD) were explored as covariates. Baseline IL-6 was chosen instead of IL- 6 from each visit because of the cause-andeffect relationship between IL- 6 and peritonitis. Patients who experienced peritonitis prior to the first PDE collection were excluded from the analysis $(n=3)$. Data were analysed using the software package Stata/SE12.0 (College Station, TX). P $<0.05$ was considered to represent statistically significant differences.

\section{Results}

\section{Patient characteristics}

The patients (biocompatible $n=42$; control $n=46$ ) were well matched for all baseline characteristics other than higher peritoneal ultrafiltration $(P=0.03)$ and lower PSTR $(P=0.006)$ at one month in the control group (Table 1$)$. The baseline characteristics of this subgroup were comparable to the original balANZ trial cohort [17].

\section{The trend of dialysate interleukin- 6 concentrations}

There was a statistically significant increase in median IL-6 concentrations over time from $7.22 \mathrm{pg} / \mathrm{mL}$ at baseline to $31.35 \mathrm{pg} / \mathrm{mL}$ at month $24(P<0.001$, Figure 1$)$. Similar trajectories were observed for the biocompatible and control groups $(P=0.68$; Figure 2$)$. There was no significant interaction between the type of PD solution received and time on PD $(P=0.09$; data not shown). PD duration remained a significant and independent predictor of IL-6 after the type of PD solutions received was added to the model (Table 2). There was no significant association between loss of residual renal function and the time trend in dialysate IL-6 concentrations $(P=0.67)$. Change in IL-6 concentration from baseline was comparable at 12 and 24 months $(P=0.12)$ but was negatively associated with baseline IL-6 levels (coefficient $-0.88, P$ $<0.001$; Additional file 1: Figure S1). A sensitivity analysis excluding an outlier produced comparable results (coefficient $-0.88, P<0.001)$. Similar results were obtained when analyses were repeated in the peritonitis-free cohort (Table 2) as well as when IL-6 AR (Additional file 1: Table S1) was analysed as the outcome.

\section{Interleukin-6 as predictor of peritoneal membrane function} Change in PSTR from baseline was significantly greater in those with higher levels of IL-6 $(P=0.004)$, who had received standard $\mathrm{PD}$ solutions $(P=0.005)$, and over longer PD duration $(P<0.001)$. The two-way interaction between PD duration and PD solution type was significant $(P=0.007)$, such that patients who received standard solutions experienced a greater increase in PSTR with longer PD duration whilst those who received biocompatible solutions maintained a relatively stable PSTR over time (Table 3; Additional file 1: Figure S2). A sensitivity analysis in the peritonitis-free cohort produced similar results (Additional file 1: Table S2) except that the effect of standard solutions on PSTR was no longer statistically significant $(P=0.06)$.

\section{Baseline interluekin- 6 as predictor of peritonitis}

Twenty-nine patients (biocompatible $\mathrm{n}=5$; control $\mathrm{n}=24$ ) experienced 45 episodes of peritonitis during the study. Peritonitis rates, expressed as episodes per patient-year, were 0.27 (95\% confidence interval [95\% CI] 0.19-0.36) overall, 0.10 (95\% CI 0.04-0.20) in the biocompatible group, and 0.41 (95\% CI 0.29-0.56) in the control group. The majority of patients experienced their first peritonitis in the first 12 months of the study (77.8\%). Baseline median dialysate IL- 6 levels in patients who experienced peritonitis against those who never experienced peritonitis were, $7.87 \mathrm{pg} / \mathrm{mL}$ and $5.75 \mathrm{pg} / \mathrm{mL}$, respectively $(P=0.15)$. According to a multivariable Cox proportional hazards model analysis, standard solution use (biocompatible solution: adjusted hazard ratio [AHR] 0.19, 95\% CI 0.07-0.50; 
Table 1 Baseline characteristics

\begin{tabular}{|c|c|c|c|c|}
\hline Characteristic & Total $(n=88)$ & Biocompatible $(n=42)$ & Control $(n=46)$ & $P$ values \\
\hline Age (yr) & $62(51-69)$ & $64(51-70)$ & $60(51-67)$ & 0.28 \\
\hline Female & $39(44.32)$ & $18(42.86)$ & $21(45.65)$ & 0.79 \\
\hline Ethnicity & & & & 0.21 \\
\hline - Caucasian & $65(73.86)$ & $34(80.95)$ & $31(67.39)$ & \\
\hline - ATSI & $3(3.41)$ & $1(2.38)$ & $2(4.35)$ & \\
\hline - Asian & $16(18.18)$ & $7(16.67)$ & $9(19.57)$ & \\
\hline$-\mathrm{MPI}$ & $4(4.55)$ & $0(0)$ & $4(8.69)$ & \\
\hline Diabetes Mellitus & $28(31.82)$ & $12(28.57)$ & $16(34.78)$ & 0.53 \\
\hline Primary renal disease & & & & 0.16 \\
\hline - Glomerulonephritis & 21(23.86) & $9(21.43)$ & 12(26.09) & \\
\hline - Diabetic nephropathy & $22(25.00)$ & $7(16.67)$ & $15(32.61)$ & \\
\hline - Hypertensive/Renovascular & $15(17.05)$ & $7(16.67)$ & $8(17.39)$ & \\
\hline - Polycystic kidney disease & $8(9.09)$ & $5(11.90)$ & $3(6.52)$ & \\
\hline - Reflux nephropathy & $4(4.55)$ & $4(9.52)$ & $0(0.00)$ & \\
\hline - Other & $18(20.45)$ & $10(23.81)$ & $8(17.39)$ & \\
\hline Body mass index $\left(\mathrm{kg} / \mathrm{m}^{2}\right)$ & & & & 0.54 \\
\hline$-<20$ & $2(2.27)$ & $0(0)$ & $2(4.35)$ & \\
\hline$-20-24.9$ & $25(28.41)$ & $13(30.95)$ & $12(26.09)$ & \\
\hline$-25-30$ & $32(36.36)$ & $16(38.10)$ & $16(34.78)$ & \\
\hline$->30$ & $29(32.95)$ & $13(30.95)$ & $16(34.78)$ & \\
\hline Haemodialysis before PD & $4(4.55)$ & $2(4.76)$ & $2(4.35)$ & 0.93 \\
\hline Initial PD modality & & & & 0.79 \\
\hline - CAPD & $81(92.05)$ & $39(92.86)$ & $42(91.30)$ & \\
\hline$-A P D$ & $7(7.95)$ & $3(7.14)$ & $4(8.70)$ & \\
\hline GFR (mL/min per $\left.1.73 \mathrm{~m}^{2}\right)$ & $7.40 \pm 2.85$ & $7.28 \pm 2.58$ & $7.51 \pm 3.09$ & 0.72 \\
\hline Urine volume (mL/day) & $1379.50(1057.5-1900)$ & $1573(1060-2059)$ & $1325(1055-1810)$ & 0.25 \\
\hline $7.5 \%$ Icodextrin use (\%) & $11(12.50)$ & $5(11.90)$ & $6(13.04)$ & 0.87 \\
\hline Ultrafiltration ${ }^{\#}(\mathrm{~mL} /$ day) & $959.50(510-1400)$ & $800(430-1300)$ & $1050(773-1500)$ & 0.03 \\
\hline Normalized ultrafiltration corrected for total glucose exposure\# & $4.08(2.21-6.04)$ & $3.18(1.78-4.19)$ & $4.59(3.17-7.45)$ & 0.002 \\
\hline Total glucose exposure (g/day) & $122.52 \pm 32.44$ & $122.38 \pm 32.35$ & $122.65 \pm 32.90$ & 0.97 \\
\hline$D: P_{C r 4 h}{ }^{*}$ & $0.64 \pm 0.10$ & $0.67 \pm 0.11$ & $0.61 \pm 0.09$ & 0.006 \\
\hline$\| \mathrm{L}-6(\mathrm{pg} / \mathrm{mL})$ & $7.22(3.93-13.71)$ & $7.61(4.66-19.98)$ & $6.42(3.29-9.63)$ & 0.17 \\
\hline IL-6 Appearance rate ${ }^{\wedge}$ & $23.19(13.66-59.35)$ & $27.14(14.12-67.27)$ & $21.90(13.66-35.01)$ & 0.45 \\
\hline
\end{tabular}

\#Measured at month $3 ;{ }^{*}$ Measured at month $1 ;{ }^{\wedge} \mathrm{IL}-6$ appearance rate $=\mathrm{IL}-6^{*}$ effluent volume/dwell time in minutes.

APD: automated peritoneal dialysis; ATSI: Aboriginal and Torres Strait Islander; CAPD: continuous ambulatory peritoneal dialysis; D:PCr4h: dialysate:plasma creatinine ratio measured at 4 hours; GFR: glomerular filtration rate; IL-6: interleukin-6; MPI: Maori \& Pacific Islander; PD: peritoneal Dialysis.

$P=0.001$ ), but not baseline IL-6 levels (AHR 1.00, 95\% CI $0.99-1.00 ; P=0.74$ ), was associated with a significantly shorter time to first peritonitis.

\section{Discussion}

The present investigation is the first study to examine the impact of biocompatible PD solution use on the utility of IL-6 as a predictor of higher PSTR and peritonitis in incident PD patients. A statistically significant increase in dialysate IL-6 concentrations with longer time on PD was observed whilst the type of PD solution (biocompatible vs. standard) and loss of residual renal function exerted no effect on its levels. Higher levels of dialysate IL- 6 and the use of standard solutions were predictive of a greater increase in PSTR. The risk of peritonitis was associated with PD solution (biocompatible solution reduced the risk) but not baseline IL-6 levels.

An increase in dialysate IL-6 levels with a longer PD duration has been inconsistently reported by several groups $[8,13,23]$ including the recently completed GLOBAL fluid study, which did not show any association between the duration of PD and dialysate IL-6 levels in incident and 


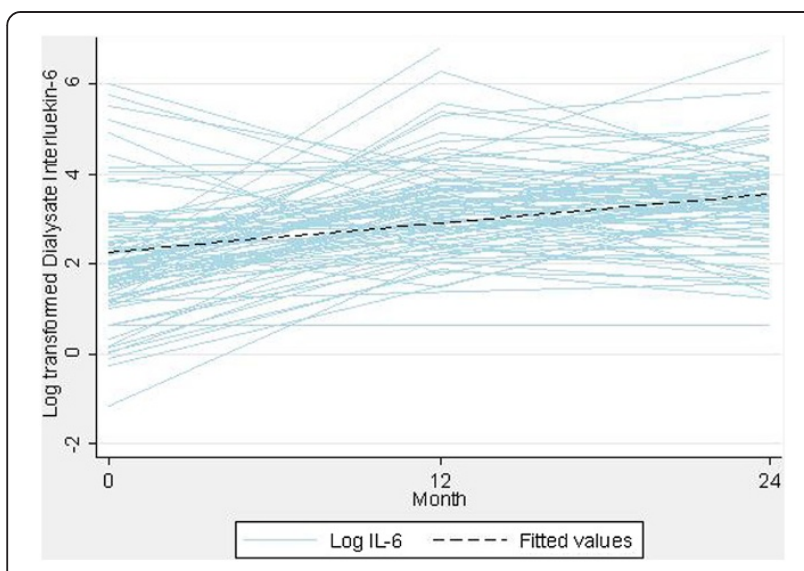

Figure 1 Overall trend of log-transformed dialysate Interleukin-6 concentrations over time in incident peritoneal dialysis patients.

prevalent PD patients [10]. While results from the GLOBAL study were strengthened by a large number of patients $(n=959)$, no data were presented pertaining to a longitudinal trend in dialysate IL-6 levels nor was there a detailed examination of the impact of biocompatible PD solution use on intraperitoneal inflammatory markers. In contrast, the extension study of the Balnet trial observed a significant increase in dialysate IL-6 levels from baseline at 24 months in both biocompatible $(143 \pm 69.6 \mathrm{pg} / \mathrm{mL}$ vs. $57.6 \pm 54.5 \mathrm{pg} / \mathrm{mL} ; P<0.001)$ and control groups $(121 \pm 69 \mathrm{pg} / \mathrm{mL}$ vs. $47 \pm 31.2 \mathrm{pg} / \mathrm{mL}$; $P<0.001$ ) [13]. There was no significant difference between the two groups $(P=0.38)$. However, the sample size was small $(n=46)$, and the potential impact of peritonitis on dialysate IL-6 levels was not separately explored. The latter factor might have been important given the discrepant number of peritonitis episodes between biocompatible and control groups (19 vs. 6 episodes, respectively), with a significantly worse peritonitis-free survival in the

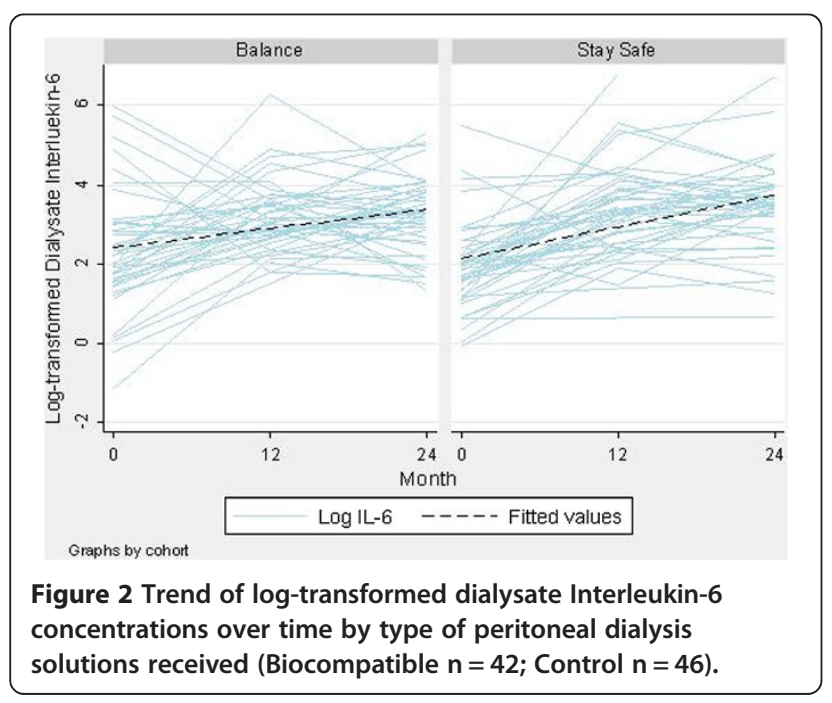

biocompatible group $(P=0.03)$. The present investigation also observed a statistically significant increase in dialysate IL-6 levels with longer PD duration with a lack of observed difference between biocompatible and standard types of PD solution. The robustness of the findings was confirmed by a sensitivity analysis performed in the peritonitis-free cohort. Nevertheless, there is conflicting information about the effect of biocompatible PD solutions on dialysate IL-6 levels. For instance, the Bicarbonate/Lactate Study group $(\mathrm{n}=92)$ reported a significant decrease in dialysate IL-6 levels at six months $(P=0.01)$ in the patients who received biocompatible solutions [24]. Although the difference in results might have stemmed from examining the biocompatible solution that contained bicarbonate-buffer, another study $(\mathrm{n}=55)$ examining bicarbonate-buffer based solution reported comparable dialysate IL- 6 levels between biocompatible and standard solution groups [14]. Therefore, the true effect of biocompatible solutions on the level of intraperitoneal inflammation, defined by IL- 6 concentrations, remains uncertain. Furthermore, the rate of loss of residual renal function exerted no significant impact on dialysate IL-6 levels. These results are supported by findings from the GLOBAL study where urine volume had no significant impact on dialysate IL-6 levels in incident PD patients $(P=0.2)$ [10].

Another important observation from the current investigation was the significant role of an individual's baseline IL-6 levels in predicting future levels. Patients with low baseline IL- 6 levels yielded the greatest increase in dialysate IL- 6 concentrations over time, whereas the opposing relationship held true for those with higher baseline IL-6 levels (Additional file 1: Figure S1). This highlights the importance of performing appropriate statistical analyses to accommodate intra-individual correlation and interindividual variation, as adopted in the present study.

Similarly, due to the presence of inter-individual variation in PSTR levels, a change in PSTR from baseline for each individual was examined. Significant associations between increasing PSTR and higher IL-6 levels, the use of standard PD solutions and longer PD duration were observed. These results are in keeping with the findings from the GLOBAL study [10]. An association between a greater increment in IL- 6 with increasing PSTR ( $r=0.306, P=0.002)$ was previously reported in a prospective 12-month longitudinal observational study $(\mathrm{n}=187)$ in incident PD patients [23]. This study allowed for patients to receive either biocompatible or standard solutions, and observed an increase in PSTR in patients receiving standard solutions over time, unlike those who were treated using biocompatible solutions who maintained a stable PSTR. However, whether PD solution type and/or dialysate IL-6 levels had differing or independent effects on PSTR was not examined. Furthermore, the 
Table 2 Multilevel linear regression to evaluate the effect of peritoneal dialysis solution type on dialysate interleukin-6 concentrations

\begin{tabular}{|c|c|c|c|c|c|c|}
\hline \multirow[b]{2}{*}{ Variable } & \multicolumn{3}{|c|}{ All patients $(n=88)$} & \multicolumn{3}{|c|}{ Peritonitis-free patients $(n=56)$} \\
\hline & Regression coefficient & Standard error & $P$ values & Regression coefficient & Standard error & $P$ values \\
\hline \multicolumn{7}{|l|}{ PD solution } \\
\hline - Control & Reference & Reference & Reference & Reference & Reference & Reference \\
\hline - Biocompatible & -0.07 & 0.17 & 0.68 & -0.14 & 0.22 & 0.54 \\
\hline Time & & & $<0.001$ & & & $<0.001$ \\
\hline - Month 0 & Reference & Reference & Reference & Reference & Reference & Reference \\
\hline - Month 12 & 1.18 & 0.14 & $<0.001$ & 1.27 & 0.17 & $<0.001$ \\
\hline - Month 24 & 1.30 & 0.17 & $<0.001$ & 1.40 & 0.22 & $<0.001$ \\
\hline
\end{tabular}

choice of therapy (biocompatible vs. standard) was at the discretion of each patient's treating physician, thereby introducing the risk of selection bias. In contrast, the cohorts from the present study were participants of a RCT with well-balanced baseline characteristics, at low risk of selection bias and with longer follow-up duration (24 months). In addition, when PSTR was re-examined in those free of peritonitis, a consistent association with dialysate IL-6 levels was observed. These associations are certainly biologically plausible as higher PSTRs are considered a functional consequence of morphological alterations at the level of the peritoneal membrane (i.e. peritoneal membrane injury), which are likely to be a result of or lead to more marked intraperitoneal inflammation.

Progressive and cumulative peritoneal injury, mediated by repeated exposures to PD solutions [2], might not only manifest with changes in the inflammatory milieu but also increase the risk of developing further problems (e.g. peritonitis) as the balance of peritoneal homeostasis and host defence are altered [25]. In this respect the IL-6

Table 3 Multilevel linear regression of change in peritoneal solute transport rate in incident peritoneal dialysis patients

\begin{tabular}{|c|c|c|c|c|c|c|}
\hline \multirow[b]{2}{*}{ Variable } & \multicolumn{3}{|c|}{ Full model } & \multicolumn{3}{|c|}{ Final model } \\
\hline & Coefficient & Standard error & $P$ values & Coefficient & Standard error & $P$ values \\
\hline $\log _{10} \| \mathrm{L}-6$ & 0.02 & 0.006 & 0.002 & 0.02 & 0.006 & 0.004 \\
\hline \multicolumn{7}{|l|}{ PD solution } \\
\hline - Control & Reference & Reference & Reference & Reference & Reference & Reference \\
\hline - Biocompatible & -0.05 & 0.02 & 0.005 & -0.05 & 0.02 & 0.005 \\
\hline \multicolumn{7}{|l|}{ PD duration } \\
\hline - 12 month & Reference & Reference & Reference & Reference & Reference & Reference \\
\hline - 24 month & 0.05 & 0.01 & $<0.001$ & 0.05 & 0.01 & $<0.001$ \\
\hline Age & 0.0002 & 0.0007 & 0.74 & & & \\
\hline Male & -0.03 & 0.02 & 0.06 & & & \\
\hline BMI (kg/m2): & & & 0.11 & & & \\
\hline$-<20$ & Reference & Reference & Reference & & & \\
\hline$-20-24.9$ & 0.03 & 0.06 & 0.59 & & & \\
\hline$-25-30$ & 0.02 & 0.06 & 0.71 & & & \\
\hline$->30$ & 0.07 & 0.06 & 0.25 & & & \\
\hline Two-way interaction (PD duration*PD solution) ${ }^{\#}$ & -0.04 & 0.01 & 0.008 & -0.04 & 0.01 & 0.007 \\
\hline Ethnicity & & & 0.31 & & & \\
\hline - Caucasian & Reference & Reference & Reference & & & \\
\hline - Asian & -0.02 & 0.02 & 0.44 & & & \\
\hline - ATSI & -0.08 & 0.05 & 0.09 & & & \\
\hline$-\mathrm{MPI}$ & 0.01 & 0.04 & 0.74 & & & \\
\hline
\end{tabular}

IL-6: Interleukin-6; PD: peritoneal dialysis; BMI: body mass index; ATSI: Aboriginal and Torres Strait Islander; MPI: Maori and Pacific Islander.

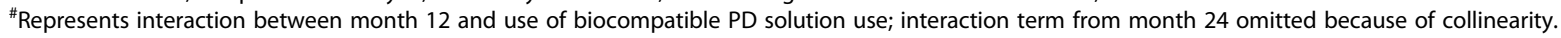


and associated signaling cascades play a key role in controlling host defence and leukocyte trafficking during infection $[6,26]$. In fact, a retrospective observational study of incident PD patients $(\mathrm{n}=31)$ receiving standard solutions reported higher baseline dialysate IL-6 concentrations in patients who developed peritonitis $(58.4 \pm 12.6 \mathrm{pg} / \mathrm{mL})$ compared to those who remained peritonitis-free $(20.3 \pm 8.7 \mathrm{pg} / \mathrm{mL}, P=0.07)$ [8]. The difference however did not reach statistical significance, and the study was limited by its small sample size and singlecentre design. Similar to their findings, a median dialysate IL-6 concentration was greater in those who experienced peritonitis in the present study, but was not statistically significant when compared to those who did not develop peritonitis. Furthermore, only the use of standard PD solutions, and not baseline IL-6, was predictive of shorter time to first peritonitis. These findings support the results from pre-clinical trials where the use of biocompatible solutions has been associated with an improvement in host cell defence [27]. The mechanisms contributing to the beneficial effect of biocompatible solutions are clearly complex but its 'protective effect' may extend beyond improved preservation of the peritoneal membrane. These beliefs have been challenged by findings from a recent observational study that reported a shorter time to first peritonitis with the use of biocompatible solutions [28]. However, this study has several limitations. These include a risk of indication bias with residual confounding, relatively low usage of biocompatible solutions in the cohort suggestive of underlying selection bias, and classifying biocompatible solutions from different manufacturers with varying GDP content in the biocompatible group which could have compromised the beneficial effect from the use of solutions that are truly low in their GDP content. On the other hand, although the balANZ trial was able to clearly demonstrate a lowered risk of peritonitis with the use of biocompatible solutions, it was one of the secondary outcomes measured during the trial $[17,20]$. Therefore, in order to evaluate the true effect of biocompatible solutions on peritonitis risk, a well designed, multicenter, multinational RCT adequately powered to examine peritonitis as a primary outcome is needed.

The present study is strengthened by its long follow-up duration in comprehensively described participants of an $\mathrm{RCT}$. It is one of the largest longitudinal studies to date exploring the utility of IL-6 in predicting important patientlevel clinical outcomes and the influence of biocompatible PD solution use. Also, given the cause-and-effect relationship between peritonitis and IL-6 levels, a sensitivity analysis including only peritonitis-free patients was performed to confirm the robustness of the findings.

However, the conclusions that can be drawn from the study are challenged by several limitations. First, the balANZ trial was a RCT with primary outcome measure of residual renal function decline, rather than a biomarker study. Therefore, the study design did not account for potential biological and pre-analytical sources of variations of IL-6, which could have affected the measured results. Second, the baseline PSTRs were not true baseline values as they were obtained at one month after study commencement. Thirdly, by selecting patients who had completed the 24-month follow-up, this might have introduced the risk of selection bias. Fourthly, some of the risk factors associated with a shorter time to peritonitis, such as educational level, [29] were not available and could not be adjusted for in the relevant analyses. And lastly, although it is one of the largest studies to date, the size is relatively small and may have increased the chance of Type II statistical error especially when comparing the outcomes between biocompatible and control groups.

\section{Conclusions}

In conclusion, dialysate IL-6 concentration increased with longer time on PD and was an independent and significant predictor of increasing PSTR. The type of PD solution did not influence dialysate IL-6 concentration levels. Standard PD solution use, but not baseline IL-6 levels, was predictive of shorter time to first peritonitis. Future studies should aim to identify the causes of higher dialysate IL-6 levels and to validate the use of IL- 6 as a potential monitoring tool with an aim to assist in risk stratifying PD patients at risk of increasing PSTR.

\section{Appendix}

\section{Collaborators (balANZ Investigators)}

Australian Centres: G Rangan, L Liew, Blacktown Hospital, Sydney (NSW); H Kulkarni, U Steinwandel, Fremantle Hospital, Fremantle (WA); B Jones, L Garvey, John Hunter Hospital, Newcastle (NSW); M G Suranyi, M Gilbert, Liverpool Hospital, Sydney (NSW); F G Brown, I Abraham, J Nandkumar Monash Medical Centre, Melbourne (VIC); A Coburn, V Bali, Princess Alexandra Hospital, Brisbane (QLD); S McDonald, S Frasca, M Hockley, C Russ, The Queen Elizabeth Hospital, Adelaide (SA); T J Elias, K Bannister, M Hockley, K Pirone, Royal Adelaide Hospital (SA); D Ranganathan, L Williams, Royal Brisbane Hospital, Brisbane (QLD); K Warr, G Smith, Perth (WA); N Boudville, S Pellicano, Sir Charles Gairdner Hospital, Perth (WA); R Langham, E O'Flaherty, St Vincents Hospital, Melbourne (VIC).

New Zealand Centres: J Schollum, L Reed, L Anderson Dunedin Hospital, Dunedin; D Voss, B Jagannathan, P Nicholls Middlemore Hospital, Auckland.

Singapore Centres- M WY Foo, CK Tam, Singapore General Hospital, Singapore; R Lee, Tang Tock Seng Hospital, Changi General Hospital, Singapore; SH Tan Kidney and Medical Clinic, Gleneagles Medical Centre, Singapore. 


\section{Additional file}

Additional file 1: Table S1. Multilevel linear regression to evaluate the effect of peritoneal dialysis solution type on dialysate interleukin-6 appearance rate. Table S2. Multilevel linear regression of change in peritoneal solute transport rate in peritonitis-free incident peritoneal dialysis patients. Figure S1. Scatter plot depicting the relationship between baseline interleukin- 6 concentrations and change in interleukin- 6 from baseline at a) Month 12 and b) Month 24. Figure S2. Trend in change in peritoneal solute transport rate (defined using 4-hour dialysate: plasma creatinine) from baseline value according to the type of peritoneal dialysis solutions received.

\section{Abbreviations}

BMl: Body mass index; CV: Coefficient of variation; GDP: Glucose degradation product; GFR: Glomerular filtration rate; IL-6: Interleukin-6; IL-6 AR: Interleukin-6 appearance rate; PD: Peritoneal dialysis; PDE: Peritoneal dialysis effluent; PSTR: Peritoneal solute transport rate; RCT: Randomized controlled trial.

\section{Competing interests}

David Johnson is a consultant for Baxter Healthcare Pty Ltd and has previously received research funds from this company. He has also received speakers' honoraria and research grants from Fresenius Medical Care. He has previously been a consultant to Gambro Pty Ltd. He is an International Society of Peritoneal Dialysis Councillor and is a current recipient of a Queensland Government Health Research Fellowship. Yeoungjee Cho is a current recipient of Australian Postgraduate Award and is a recipient of 2012 Jacquot Research Entry Scholarship. Carmel Hawley has received research grants from Baxter Healthcare Pty Ltd and Gambro Pty Ltd, and has been a consultant to Fresenius Medical care. Margaret Clarke is an employee of Fresenius Medical Care. Remaining authors declare no conflicts of interest.

\section{Authors' contributions}

The study was conceived, designed, and supervised by authors YC, DWJ, CH, NT, DV (non-Fresenius employee). MC was involved in the acquisition of the data. YC wrote the first draft of the manuscript, subsequent drafts were reviewed and approved by YC, DWJ, DV, CH, EP, MC, and NT.

\section{Acknowledgement}

The invaluable assistance with measurement of dialysate IL-6 levels provided by Dr. Sabine Lange (employee of Meso-scale discovery, Gaithusburg, MD, USA) is gratefully acknowledged. This biomarker sub-study of the balANZ trial was funded by the Fresenius Research Grant to fund the purchase of kits to measure dialysate IL-6 levels. The funding body served no role in study conception, data analysis or the preparation of the manuscript.

\section{Author details}

'Department of Renal Medicine, Princess Alexandra Hospital, Brisbane, Australia. ${ }^{2}$ School of Medicine, University of Queensland, Brisbane, Australia. ${ }^{3}$ Translational Research Institute, University of Queensland, Brisbane, Australia. ${ }^{4}$ Fresenius Medical Care, Sydney, Australia. ${ }^{5}$ Institute of Translation, Innovation, Methodology and Engagement, Cardiff University School of Medicine, Cardiff, UK.

Received: 23 October 2013 Accepted: 7 January 2014

Published: 10 January 2014

\section{References}

1. Dobbie JW: Morphology of the peritoneum in CAPD. Blood Purif 1989, 7(2-3):74-85.

2. Williams JD, Craig KJ, Topley N, Von Ruhland C, Fallon M, Newman GR, Mackenzie RK, Williams GT: Morphologic changes in the peritoneal membrane of patients with renal disease. J Am Soc Nephrol 2002, 13(2):470-479.

3. Davies SJ, Phillips L, Russell GI: Peritoneal solute transport predicts survival on CAPD independently of residual renal function. Nephrol Dial Transplant 1998, 13(4):962-968.

4. Fried L: Higher membrane permeability predicts poorer patient survival. Perit Dial Int 1997, 17(4):387-389.
5. Rumpsfeld M, McDonald SP, Johnson DW: Higher peritoneal transport status is associated with higher mortality and technique failure in the Australian and New Zealand peritoneal dialysis patient populations. J Am Soc Nephrol 2006, 17(1):271-278.

6. Hurst SM, Wilkinson TS, McLoughlin RM, Jones S, Horiuchi S, Yamamoto N, Rose-John S, Fuller GM, Topley N, Jones SA: II-6 and its soluble receptor orchestrate a temporal switch in the pattern of leukocyte recruitment seen during acute inflammation. Immunity 2001, 14(6):705-714.

7. Witowski J, Jorres A, Coles GA, Williams JD, Topley N: Superinduction of IL-6 synthesis in human peritoneal mesothelial cells is related to the induction and stabilization of IL-6 mRNA. Kidney Int 1996, 50(4):1212-1223.

8. Pecoits-Filho RCM, Stenvinkel P, LIndholm B, Heimburger O: Systemic and intraperitoneal interleukin- 6 system during the first year of peritoneal dialysis. Perit Dial Int 2006, 26:53-63.

9. Oh KH, Jung JY, Yoon MO, Song A, Lee H, Ro H, Hwang YH, Kim DK, Margetts $P$, Ahn C: Intra-peritoneal interleukin- 6 system is a potent determinant of the baseline peritoneal solute transport in incident peritoneal dialysis patients. Nephrol Dial Transplant 2010, 25(5):1639-1646.

10. Lambie M, Chess J, Donovan KL, Kim YL, Do JY, Lee HB, Noh H, Williams PF, Williams AJ, Davison S, et al: Independent Effects of Systemic and Peritoneal Inflammation on Peritoneal Dialysis Survival. J Am Soc Nephrol 2013, 24:2071-2080.

11. Ayuzawa N, Ishibashi Y, Takazawa Y, Kume H, Fujita T: Peritoneal morphology after long-term peritoneal dialysis with biocompatible fluid: recent clinical practice in Japan. Perit Dial Int 2012, 32(2):159-167.

12. Mortier S, Faict D, Schalkwijk CG, Lameire NH, De Vriese AS: Long-term exposure to new peritoneal dialysis solutions: Effects on the peritoneal membrane. Kidney Int 2004, 66(3):1257-1265.

13. Kim S, Oh KH, Oh J, Kim SJ, Chung W, Song YR, Na KY, Oh YK, Ahn C, Kim $\mathrm{SG}$, et al: Biocompatible peritoneal dialysis solution preserves residual renal function. Am J Nephrol 2012, 36(4):305-316.

14. Weiss L, Stegmayr B, Malmsten G, Tejde M, Hadimeri H, Siegert CE, Ahlmen $J$, Larsson R, Ingman B, Simonsen $O$, et al: Biocompatibility and tolerability of a purely bicarbonate-buffered peritoneal dialysis solution. Perit Dial Int 2009, 29(6):647-655.

15. Lai KN, Lam MF, Leung JC, Chan LY, Lam CW, Chan IH, Chan HW, Li CS, Wong SS, Ho YW, et al: A study of the clinical and biochemical profile of peritoneal dialysis fluid low in glucose degradation products. Perit Dial Int 2012, 32(3):280-291.

16. Kim S, Oh J, Chung W, Ahn C, Kim SG, Oh KH: Benefits of biocompatible PD fluid for preservation of residual renal function in incident CAPD patients: a 1-year study. Nephrol Dial Transplant 2009, 24(9):2899-2908.

17. Johnson DW, Brown FG, Clarke M, Boudville N, Elias TJ, Foo MW, Jones B, Kulkarni H, Langham R, Ranganathan D, et al: Effects of Biocompatible versus Standard Fluid on Peritoneal Dialysis Outcomes. J Am Soc Nephrol 2012, 23(6):1097-1107.

18. Johnson DW, Clarke M, Wilson V, Woods F, Brown FG: Rationale and design of the balANZ trial: a randomised controlled trial of low GDP, neutral $\mathrm{pH}$ versus standard peritoneal dialysis solution for the preservation of residual renal function. BMC Nephrol 2010, 11:25.

19. Johnson DW, Brown FG, Clarke M, Boudville N, Elias TJ, Foo MW, Jones B, Kulkarni $H$, Langham R, Ranganathan D, et al: The effect of low glucose degradation product, neutral $\mathrm{pH}$ versus standard peritoneal dialysis solutions on peritoneal membrane function: the balANZ trial. Nephrol Dial Transplant 2012.

20. Johnson DW, Brown FG, Clarke M, Boudville N, Elias TJ, Foo MW, Jones B, Kulkarni H, Langham R, Ranganathan D, et al: The Effects of Biocompatible Compared with Standard Peritoneal Dialysis Solutions on Peritonitis Microbiology, Treatment, and Outcomes: the balANZ Trial. Perit Dial Int 2012, 32(5):497-506

21. Rumpsfeld M, McDonald SP, Purdie DM, Collins J, Johnson DW: Predictors of baseline peritoneal transport status in Australian and New Zealand peritoneal dialysis patients. Am J Kidney Dis 2004, 43(3):492-501.

22. Davies SJ: Mitigating peritoneal membrane characteristics in modern peritoneal dialysis therapy. Kidney Int Supp/ 2006, 103:S76-83.

23. Cho JH, Hur IK, Kim CD, Park SH, Ryu HM, Yook JM, Choi JY, Choi HJ, Park $J W$, Do JY, et al: Impact of systemic and local peritoneal inflammation on peritoneal solute transport rate in new peritoneal dialysis patients: a 1-year prospective study. Nephrol Dial Transplant 2010, 25(6):1964-1973.

24. Cooker LA LP, Holmes CJ, Jones S, Topley N, on behalf of the Bicarbonate/ Lactate study group: Interleukin-6 levels decrease in effluent from 
patients dialyzed with bicarbonate/lactate-based peritoneal dialysis solutions. Perit Dial Int 2001, 21(Supplement 3):S102-S107.

25. Yung S, Chan TM: Pathophysiological changes to the peritoneal membrane during PD-related peritonitis: the role of mesothelial cells. Mediators Inflamm 2012, 2012:484167.

26. McLoughlin RM, Witowski J, Robson RL, Wilkinson TS, Hurst SM, Williams AS, Williams JD, Rose-John S, Jones SA, Topley N: Interplay between IFN-gamma and IL-6 signaling governs neutrophil trafficking and apoptosis during acute inflammation. J Clin Invest 2003, 112(4):598-607.

27. Mortier S, De Vriese AS, McLoughlin RM, Topley N, Schaub TP, Passlick-Deetjen J, Lameire NH: Effects of conventional and new peritoneal dialysis fluids on leukocyte recruitment in the rat peritoneal membrane. J Am Soc Nephrol 2003, 14(5):1296-1306.

28. Cho Y, Badve SV, Hawley CM, McDonald SP, Brown FG, Boudville N, Bannister KM, Clayton PA, Johnson DW: Association of biocompatible peritoneal dialysis solutions with peritonitis risk, treatment, and outcomes. Clin J Am Soc Nephrol 2013, 8(9):1556-1563.

29. Martin LC, Caramori JC, Fernandes N, Divino-Filho JC, Pecoits-Filho R, Barretti P, Brazilian Peritoneal Dialysis Multicenter Study BG: Geographic and educational factors and risk of the first peritonitis episode in Brazilian Peritoneal Dialysis study (BRAZPD) patients. Clin J Am Soc Nephrol 2011, 6(8):1944-1951.

doi:10.1186/1471-2369-15-8

Cite this article as: Cho et al.: Dialysate interleukin- 6 predicts increasing peritoneal solute transport rate in incident peritoneal dialysis patients. BMC Nephrology 2014 15:8.

\section{Submit your next manuscript to BioMed Central and take full advantage of:}

- Convenient online submission

- Thorough peer review

- No space constraints or color figure charges

- Immediate publication on acceptance

- Inclusion in PubMed, CAS, Scopus and Google Scholar

- Research which is freely available for redistribution 